\title{
An Empirical Study on the Effect of Implementing Formative Assessment in the Preparatory Education of Undergraduate Foreign Students Founded by the Chinese Government Scholarship
}

\author{
Hongjian Xu \\ School of Preparatory Education, Faculty of International Education of Chinese Language, Beijing \\ Language and Culture University, Beijing, China \\ ye333xu@163.com
}

Keywords: Formative assessment; Preparatory education; TCSL; Effect of teaching

\begin{abstract}
In order to improve the quality of the Preparatory Education of Undergraduate Foreign Students Founded by the Chinese Government Scholarship, the author wants to compare the effect of formative assessment and the traditional summative assessment. This study experimentally investigated the zero-based preparatory foreign students in our college, the results of the study showed that the examination results of the experimental group evaluated with formative assessment are significantly better than those of the control group evaluated with traditional summative assessment. It shows that formative assessment is a better way to evaluate the preparatory education in China.
\end{abstract}

\section{中国政府奖学金本科来华留学生预科教育形成性 评价效果实证研究}

\author{
许宏鉴 \\ 北京语言大学 汉语国际教育学部 预科教育学院，中国 北京 100083 \\ ye333xu@163.com
}

摘要: 为了提高中国政府奖学金本科来华留学生预科教育的教学质量, 作者欲考察形成性评 价是否比传统的终结性评价对学生学习成绩有更好的效果。本文对本学院的零起点预科留学 生进行了教学实验研究, 研究结果发现, 采用形成性评价教学的实验班的预科结业考试成绩 显著好于采用传统终结性评价的对照班。说明形成性评价对于来华预科教育来说是一种更好 的评价方式。

关键词：形成性评价；预科教学；对外汉语教学；教学效果

\section{1. 引言}

随着中国成为世界重要的留学目的地国家，来华留学生预科教育越来越重要。来华留学生预 科教育是指来中国接受高等教育的留学生为了进入大学学习专业知识, 所接受的汉语言和相 关专业基础知识的教育。其中中国政府奖学金本科来华留学生预科教育是指国家留学基金委 委托十余所国内高校对即将进入大学进行本科学习的留学生进行的预科教育。因为有国家政 策的支持, 对外汉语教学实力雄厚的高校的参与, 使得来华预科生教育发展迅速, 也取得了 初步的成果。

虽然汉语国际传播不断深化，但是汉语作为外语在世界上的位置依然不乐观，汉语的普及率 还是非常低, 来中国的预科留学生大部分没有任何汉语基础的, 或者只学过最基础的汉语。 在中国接受短短一年的预科教育, 就要进入中国大学和中国学生一起学习专业, 除去假期和 结业考试, 只有最多 8 个月的正式教学时间。与中国到英语国家接受预科教育的留学生相比, 
就更能看出学习时间的差距。一年时间从零起点培养到入大学学习专业的标准, 预科学生不 仅要学习汉语知识, 还要接受预科结业考试内容和专业学习所需技能和基础知识的教学和培 训, 学习时间短, 任务重。

来华预科留学生绝大多数都是刚刚高中毕业的未成年人, 离开自己的国家来到国外独立生活、 学习, 离开父母、家人的陪伴和督促, 很有可能会放松对自己的要求; 有的学生没有养成良 好的学习习惯差, 和生活规律散漫, 需要教师帮助他们培养良好的学习和生活习惯。

预科教育的教、学压力都很大。目前国内有 10 所大学同时接受国家留学基金委的委托开展来 华留学生预科教育, 各个大学之间在客观上也存在相互竞争的关系。来华预科留学生都是要 在一年的预科学习以后进入通过留学基金委预科结业考试才能升入大学学习, 而如果不能通 过考试则要延期一年预科学习，如果再不能通过就要被取消进入大学学习的资格。

预科教育的这些特点要求我们对学生进行更严格的全方位教育, 并且在学生学习的全过程对 学生的学习、生活情况进行关注, 并及时对教、学中出现的问题和情况进行完善和改进, 查 漏补缺, 以免积重难返。有学者提出了对预科留学生进行素质教育的必要性 (董琳莉、刘徳联, 2009)。这也要求我们及时发现学生学习中的思想波动和问题及时解决。

形成性评价被提出以后得到了充分重视, Black \& Wiliam（1998）指出：教师对学习者的学习 和学习者对自己的学习进行评价的活动, 为教学的改进提供信息, 当这些信息被用于调整教 学时就称为 “形成性评价”。HarlenW \&JamesM (1997) 强调形成性评价的关键是认清学生现 有水平和学习目标之间的差距。在国内, 较有权威性的阐述出自于国家教育部新制订的《英 语课程标准》 (2001) 和教育部高教司提出的的《大学英语课程教学要求》 (2007)。形成性评 价是不同于终结性评价的评价形式, 是一种更注重过程和反馈的评价形式, 通过对学生学习 过程进行实时监控, 即时对学生的问题和薄弱环节进行反馈, 并给教师提供根据学生学习情 况和出现问题改进的依据。形成性评价是综合性评价, 不仅对学生的学习进行评价还可以对 学生的全面表现进行评价。

形成性评价在教学中有很多的优势, 可以保证教学中有效信息的传递; 有利于因材施教; 指 导教师不断完善教学工作; 有助于教师丰富经验, 提高教学成效 (宋海燕, 2008)。形成性评价 还可以有效提高学生学习主动性 (蒋宇红与周红, 2010), 更能有效地促进学生英语学习策略 的应用, 从而更快地提高学生英语学习能力（王京华等，2006)。

在中国大学英语外语学习的研究中, 蒋宇红与周红 (1010) 对所教授的两个平行班进行了 16 周试验研究, 研究结果显示采用形成性评价的班级学生学习成绩高于采用传统的终结性评价 的班级的学生, 统计检验呈边缘显著。王京华等 (2006) 对河北大学非英语专业的八个平行 班进行了长达两年的实验研究, 发现采用形成性评价的学生的英语水平显著高于采用单一终 结性评价的学生。李燕 (2009) 研究了形成性课堂评估在对外汉语教学中实施, 提出了形成 教、学、评三位一体的完整体系, 并提出了评估具体实施方法和保证形成性课堂评估实施效 果的因素。汪冀冀 (2013) 对形成性评价在汉语任务型口语教学中的应用进行了研究, 提出 了针对该课程的形成性评价的操作模式和理论。宋燕 (2014) 研究了对 CIEE 项目四名学生进 行的形成性评价教学, 并对过程进行严格控制和及时反馈, 并对其进行了详细描写和分析, 构建了形成性评估操作框架。虽然已经有了一些研究成果, 但是已有的对外汉语教学形成性 评价的实证性研究比较少, 关于来华留学生预科教育的形成性评价研究更是没有看到。

\section{2. 实验研究}

\section{1. 研究问题}

采用形成性评价对学生的学习成绩有何影响?

\section{2. 实验背景}

实验课以本校预科教育学院零起点 4 个班级为实验班级, 共 64 名学生, 来自 39 个国家, 其 中男生 40 名, 女生 24 名。试验时间为一学年, 共 30 教学周, 周平均课时为 30 节。对照班 级为上年度课程设置、学时相同的 3 个零起点班的 65 名学生, 来自 41 个国家, 其中男生 35 
名, 女生 30 名。连续两年的教师保持不变, 学生水平都是零起点, 学生年龄都是 18-25 岁之 间。

2. 3. 测量工具

使用统计工具 SPSS 对两学年学生经过一学年学习参加国家留学基金委预科结业考试的成绩 进行分析比较, 观察两个学年学生的预科结业考试成绩是否存在差异。该考试作为国家级考 试, 具有较高的权威性和科学性, 难度具有稳定性（王佶旻等, 2014)。

2.4. 教学实验设计和形成性评价过程

在对形成性评价的理论原理和具体操作方法进行研究之后, 我们结合北京语言大学预科教育 学院的教学实际和现实条件, 在教学中实施了形成性评价。本评价本着开放性的原则, 不以 学生学习成绩为唯一的评价内容, 而是对学生学习、生活和各种活动中的表现都进行记录, 对学生进行全面的评价。不以考试为唯一评价形式, 而是采用各种方式, 全方位掌握学生的 学习、生活和思想状况, 及时发现问题, 解决问题。本实验研究从三个方面对学生进行形成 性评价并进行反馈。(见图表 1-1)

2. 4.1 学习过程评价及反馈

对预科生来说, 学习成绩对他们来说非常重要, 因此及时掌握他们的学习情况和出现的问题 非常重要, 对学生学习过程进行形成性评价能够在第一时间发现问题, 解决问题。因此我们 在学生学习全过程采取多种形式的过程评价, 并及时反馈。

(1) 课堂听写、作业

在教学中, 我们采用了听写和留作业检查的方式。听写的内容包括拼音、生词和课文中的句 子，作业则包括重复性的练习和作文、口头报告等活用练习。

(2) 周考、月考

我们在教学中每周进行周考, 每月进行月考, 周考和月考的出题体现了激励性和真实性原则。

(3) 学习者学习档案

教师会把学生平时所做的语言实践任务的文字资料, 制作 PPT 或者视频都保存在电脑里, 作 为学生评价的一个部分。

(4) 教师谈话

教师经常利用课间、课下时间跟学生进行交流, 通过有针对性的对话对学生的学习情况进行 评估; 通过学生的自我评价, 了解学生学习情况, 学习的难点、问题。

对学习过程评价结果的反馈:

对于在学习过程评价中发现的问题进行分析总结, 对于普遍性的问题, 研究有针对性的改进 教学的方案; 对于个别学生特殊的问题, 对其进行个别的辅导。要求学生使用纠错本, 把作 业、考试中出现的错误都记录下来, 作为反复学习和复习的重点。对于谈话中发现的学生的 问题, 采取有效方法予以解决。对学习过程的评价可以发现学习困难学生, 教师会对学习困 难学生给与特别的关注和帮助。

2. 4.2 学习习惯、态度评价及反馈

我们为学生印制了每一课的预习单, 根据学生对预习单的反馈, 获知学生预习情况, 由此来 观察学生是否具有课前预习的学习习惯。观察学生是否具有记课堂笔记、课下向老师提问和 抓住机会练习口语的学习习惯。

教师还会观察学生出勤、作业完成、上课回答问题、上课是否集中精力听讲等情况, 对学生 的学习态度进行时日常的评价。

对学习习惯和态度的评价结果的反馈:

把观察到的好的学习习惯在班级中进行介绍, 建议同学们根据自己的实际情况为己所用, 帮 助那些没有形成良好的学习习惯的同学建立适合自己的学习习惯。

对于发现的存在学习态度问题的学生, 要进行谈话了解情况, 若确为主观态度有问题, 学院 将会根据相关规定进行警告和进一步的处理。

2. 4.3 综合表现评价及反馈 
教师还对学生是否积极主动参加学校、学院和班级组织的各种活动进行观察和记录, 并进行 计量统计。

对综合表现评价结果的反馈:

根据学生在每次活动中的表现的记录和评价, 对于积极参加活动的同学给与表扬, 对于在活 动中发现有问题问题和表现不积极、缺乏集体意识的同学进行谈话, 帮助他们更好地融入集 体, 也为将来他们的大学专业学习和生活做好准备。学期末和学年末会评选出优秀表现奖, 综合表现的评价结果是评奖的重要依据。

\section{表 1.1 形成性评价内容}

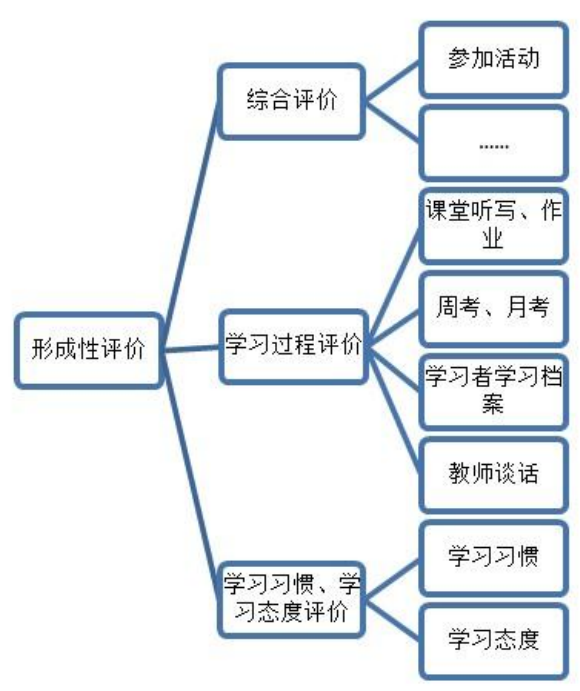

\section{3. 数据分析及讨论}

表 2-1 独立样本 T 检验

\begin{tabular}{|c|c|c|c|c|c|}
\hline 班级 & 学生数 & 平均分 & 标准差 & $\mathrm{T}$ 值 & $\mathrm{P}$ 值 \\
\hline 实验班 & 54 & 99.30 & 14.144 & \multirow{2}{*}{2.876} & .005 \\
\hline 对照班 & 48 & 88.48 & 22.385 & & \\
\hline
\end{tabular}

从表 2-1, 我们可以看到, 实验班的平均成绩为 99.3, 高于对照班的 88.48, 实验班与对照 班的预科结业考试成绩存在显著差异 $(\mathrm{T}=2.876, \mathrm{P}=0.005)$ 。这说明进行形成性评价的教学模 式对于学生的学习成绩的促进作用大于传统的终结性评价的教育模式, 这与蒋宇红等 (2010) 对中国大学英语外语学习者的研究结果一致。该研究中的统计结果为差异边缘显著 ( $\mathrm{P}=0.052)$, 作者认为是由于研究周期不够长, 如果延长试验周期, 实验班的学习成绩的优势就会体现得 更加明显。本研究被试是来华预科留学生, 结果显示形成性评价对来华预科留学生同样具有 良好的效果, 而且显著程度更加明显 ( $\mathrm{p}=0.005)$ 。这可能是与来华预科留学生的特点有关, 他们都是刚刚高中毕业尚未进入大学学习, 来到遥远的中国, 难免会有文化休克和语言休克, 老师的及时帮助可以使他们尽早度过这个休克期。没有了家人的约束, 学生们具有放松的心 态, 而预科教育又恰恰是学习强度很高的 “应试教育”, 中外教育理念、教育方式的不同, 也 会让学生不适应。因此, 及时发现学生的问题, 并且及时帮助学生建立适合自己和汉语学习、 预科学习的学习习惯都对他们能否很好地完成预科学业起着重要作用。这与王京华 (2006) 年的研究结果一致。该研究的研究周期是两年, 这说明足够长的教学时间, 是形成性评价起 作用的重要保证。因为形成性评价建构主义为理论基础, 建构主义理论认为学习者通过新旧 经验间双向的相互作用而建构自己的经验体系和认识他们自己的世界 (Von Glasersfeld, 1995), 
这需要比较长的过程。另外, 形成性评价帮助学生形成良好的学习习惯和态度, 做到这样的 转变, 也需要足够长的时间才能实现。

\section{4. 对来华留学生预科教育教学的建议}

预科教学不同于普通的留学生教学, 在学习结束时要对学生的学习进行严格的考核, 如果不 能顺利升入大学进行专业学习, 会对学生本人, 两国政府都是很大的损失。我们的研究显示, 形成性评价是一种非常有效的评价模式, 采用这样的评价方式会对学生的学习成绩、良好学 习习惯的养成和学习态度的树立都有非常大的帮助, 可以让刚刚走出高中校门来到中国的留 学生尽快适应在中国的生活和学习, 适应汉语学习的特点和预科学习的模式。研究证明, 形 成性评价的优势需要长时间才能发挥出来, 因此在学生一入校就应该采用形成性评价的教学 方式, 并且在整个学习过程中始终贯彻这样的评价模式。形成性评价的内容应该是全面的, 不仅对学生的学习进行评价, 还应该对学生的学习习惯、学习态度和综合表现进行评价。因 为预科留学生不同于普通的留学生, 他们将来是要在中国上大学, 和中国学生同堂进行专业 学习, 因此良好的学习习惯、学习态度的培养, 对于学生日后的专业学习至关重要。预科学 生年龄都比较小, 人生观、价值观多未形成, 因此帮助学生树立集体主义精神, 对于学生在 将来的大学学习和以后的发展都具有重要的意义。采用形成性评价能否取得预期效果, 对评 价结果的利用和及时有效反馈是非常重要的组成部分。形成性评价的重要特征就是过程评价, 通过在学生学习过程中情况进行评价并及时给与反馈, 帮助学生及时修正自己的行为, 这是 最关键的。

\section{5. 结论}

来华留学生预科教育是新时期的重要课题, 怎么能在短短一年的时间里使学生掌握必需的汉 语技能和相关的专业背景知识, 是我们应该深入研究的。采用形成性评价的教学模式在实验 研究和教学实践中都取得了可喜的成果, 因此我们应该把这样的成果应用在我们的教学中去, 在进行形成性评价教学的过程中, 不仅要采用形成性评价的形式, 更应该了解和贯彻形成性 评价的实质：在过程中评价, 及时进行反馈, 让学生自我建构自己的知识体系。 本研究因为是基于学院正常教学, 在同一学年中难以设立对照组, 因此以上一学年的学生作 为对照组。但是本实验中实验组和对照组的水平都是零起点, 学生初始水平相同, 两个学年 的课程设置、学时相同, 任课教师也相同, 因此在一定程度上可以作为研究对比的对象。以 后有条件, 应该在设置更为科学的实验组和对照组的情况下进行, 应该可以获得更加可靠的 结论。

\section{6. 致谢}

本研究得到北京语言大学 2015 年度院级项目（15YJ080404）的支持。

\section{参考文献}

[1] Black P J.\&Wiliam D. Assessment and classroom learning [J]. Assessment in Education. 1998 (1).

[2] Harlen W.\& M. James. Assessment and Learning:Differences and Relationships between Formative and SummativeAssessment in Education. [M]. Carfax Publishing Ltd.1997, Oxfordshire.

[3] Glasersfeld, E. V. Radical constructivism: A way of knowing and learning.[M]. Falmer Press. 1995, London. 
[4] 中华人民共和国教育部. 英语课程标准(全日制义务教育, 普通高级中学版)(实验稿)[S]. 北 京师范大学出版社. 2001, 北京.

[5] 教育部高等教育司. 大学英语课程教学要求 $[S]$. 外语教学与研究出版社. 2007, 北京.

[6] 董琳莉, 刘徳联.素质教育一留学生预科教育的新课题[J]. 海外华文教育, 2009, (1): 9-17.

[7] 宋海燕. 形成性评价及其在对外汉语教学中的应用[J]. 教科文汇, 2008, (8): 99.

[8] 蒋宇红, 周红. 大学英语采用形成性评价促进学生自主学习的实证研究[J]. 北京第二外国 语学院学报, 2010 (2): 69-74.

[9] 王京华.形成性评价对英语学习策略影响的实验研究[J]. 河北大学学报 (哲学社会科学版), 2006(4): 121-125.

[10]李燕. 对外汉语教学形成性课堂评估探析 [J]. 鲁东大学学报(哲学社会科学版), 2009, 95):30-33.

[11] 汪冀冀. 汉语任务型口语教学中形成性评价的应用研究[D]. 硕士论文, 华东师范大学, 中 国, 2013.

[12] 宋燕. 形成性评估在对外汉语教学中的应用[D]. 硕士论文, 华东师范大学, 中国,2014.

[13]王佶旻, 郭树军, 黄理兵等. 中国政府奖学金本科来华留学生预科教育“基础汉语考试”试 卷设计与质量分析 [J]. 中国考试, 2014(2): 19-25.

\section{Acknowledgement}

This research was financially supported by the 2015 Research Project of courtyard's level item in Beijing Language and Culture University (15YJ080404)

\section{References}

[1] Black P J.\&Wiliam D. Assessment and Classroom Learning [J]. Assessment in Education. 1998 (1).

[2] Harlen W.\& M. James. Assessment and Learning:Differences and Relationships between Formative and Summative Assessment in Education. [M] (Carfax Publishing Ltd, the United Kingdom1997).

[3] Glasersfeld, E. V. Radical constructivism: A way of knowing and learning. [M] (Falmer Press. the United Kingdom1995).

[4] The Ministry of education of the people's Republic of China. English Curriculum Standards (full time compulsory education, general senior middle school edition) (experimental version) [S]. Beijing Normal University press. 2001. Beijing. (In Chinese)

[5] Department of higher education of the Ministry of education. College English teaching requirements [S]. Foreign language teaching and Research Press. 2007, Beijing. (In Chinese)

[6] L.L. Dong and D.L. Liu. Quality Education -- a New Research Topic of Overseas Students Preparatory Education [J]. Overseas Chinese education, 2009, (1) p.9-17. (In Chinese)

[7] H.Y. Song. Formative Assessment and Its Application in Teaching Chinese as a Foreign Language [J]. The Science Education Article Collects, 2008, (8) p.99. (In Chinese)

[8] Y.H. Jiang and H. Zhou. An Empirical Study on the Promotion of Students' Autonomous Learning by Formative Assessment in College English [J]. Journal Beijing International Studies University, 2010 (2) p.69-74. (In Chinese) 
[9] J.H. Wang. An Experimental Study on the Influence of Formative Assessment on English Learning Strategies [J]. Hebei University Journal (Philosophy and social Sciences Edition), 2006(4) p.121-125. (In Chinese)

[10] Y. Li. Study on the Formative Assessment for the Classes in Teaching Chinese as a Second Language [J]. Ludong University Journal (Philosophy and social Sciences Edition), 2009, (5) p.30-33. (In Chinese)

[11] J.J. Wang. A Formative Assessment on Task-based Teaching Method for Oral Chinese Class [D]. (MS., East China Normal University, China 2013) (In Chinese)

[12] Y. Song. Application of Formative Assessment to Teaching Chinese as a Second Language-a Case Study of CIEE [D].(MS., East China Normal University, China 2014) (In Chinese)

[13]J.M. Wang, S.J. Guo, L.B. Huang, et al. Design and Quality Analysis on Chinese Proficiency Test (Basic) for the Preparatory Education of Undergraduate Foreign Students Founded by the Chinese Government Scholarship [J]. China Examinations, 2014(2)p.19-25. (In Chinese)

作者简介: 许宏鉴 (1978-), 男, 北京语言大学汉语国际教育学部预科教育学院, 讲师, 研 究方向为对外汉语教学、汉语第二语言习得, Email:ye333xu@163.com 